\section{Orgasmus löscht das Kurzzeitgedächtnis}

Für eine 54-jährige Frau folgte auf den Orgasmus der Schock: Plötzlich waren alle Erinnerungen aus den letzten 24 Stunden gelöscht. Auch was in den 20 bis 40 Minuten nach dem sexuellen Höhepunkt geschah, konnte sich die Frau nicht merken, berichtete der Ehemann in der Notaufnahme. Die klinische Untersuchung und die Anamnese ergaben keinerlei Auffälligkeiten. Weder hatte die Frau zuvor schon vergleichbare amnestische Ereignisse erlebt, noch hatte sie Zeichen von transienten ischämischen Attacken (TIA) bemerkt. Das MRT inklusive Angiografie war unauffällig. Die Diagnose lautete schließlich transiente globale Amnesie (TGA).

Diese Amnesieform tritt jährlich bei drei bis fünf von 100000 Personen auf, bei über 50-Jährigen liegt die Inzidenz mit etwa 23 pro 100000 deutlich höher. Für Ärzte ist vor allem wichtig, ernste neurologische Ursachen auszuschließen. Die Autoren raten jedoch, auf eine weiterführende Diagnostik zu verzichten, wenn die Patienten keine zusätzlichen neurologischen Symptome haben, die Amnesie sich rasch zurückbildet und keine Risikofaktoren für TIA und Schlaganfall vorliegen.

J Emerg Med 2011; 41:257-260.

\section{GALLENSÄUREN ALS INDIKATOR}

\section{Darmbakterien beeinflussen Statintherapie}

Wie gut eine Behandlung mit Statinen anschlägt, hängt nicht zuletzt von der Darmflora ab. Wissenschaftler von der Duke University in Durham, North Carolina, analysierten Plasmaproben von 944 Probanden, die ein Cholesterin zwischen 160 und 400 $\mathrm{mg} / \mathrm{dl}$ aufwiesen und sechs Wochen lang täglich 40 mg Simvastatin einnahmen. Die Forscher verglichen 24 Probanden, deren LDL-Cholesterin unter der Statintherapie stark sank, mit 24 Teilnehmern, die nur wenig auf Simvastatin reagierten. Dabei zeigte sich, dass ein Zusammenhang zwischen den prätherapeutischen Plasmaspiegeln von sekundären Gallensäuren sowie Koprosterin und dem Therapieeffekt bestand: je konzentrierter die Säuren, desto ausgeprägter die LDL-Reduktion. Diese Gallensäuren werden ebenso wie Koprosterin von Darmbakterien produziert. Möglicherweise optimiert eine entsprechende Diät das Ansprechen auf Statine. Metabolische Profile schätzen.

PLOS ONE 6(10): e25482. doi:10.1371/journal. pone.0025482 könnten helfen, den Therapieerfolg abzu-

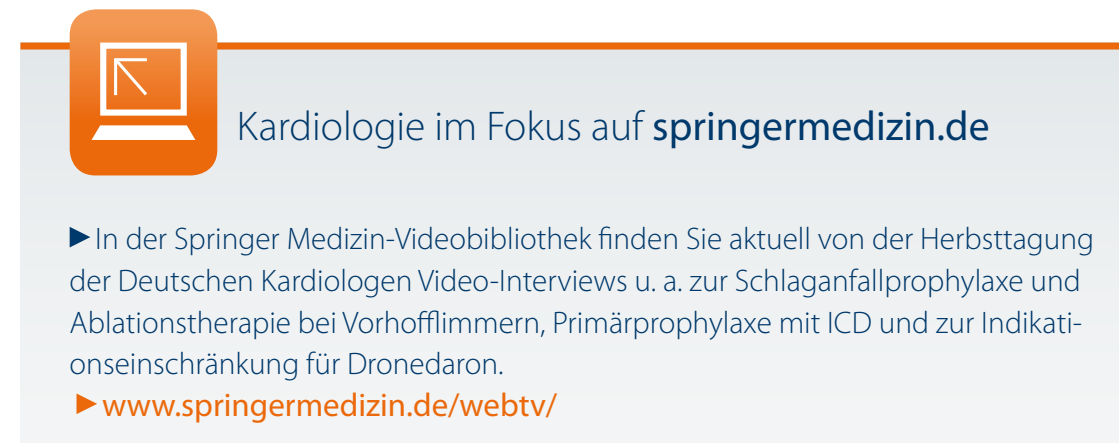

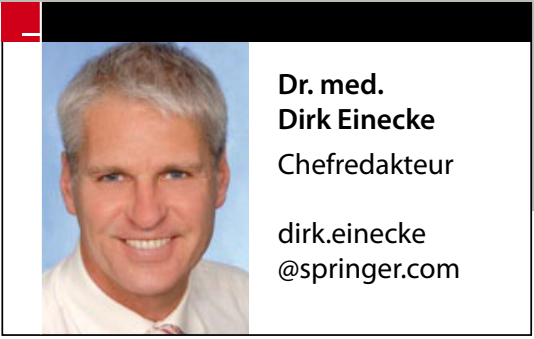

\section{PROGNOSEFAKTOR NACH SCHLAGANFALL}

\section{Schlägt der Patient die Beine übereinander?}

\begin{abstract}
Wer nach einem schweren Schlaganfall schon früh die Beine übereinanderschlagen kann, hat offenbar deutlich bessere Überlebenschancen, weniger neurologische Schäden und mehr Aussicht auf ein unabhängiges Leben als ein Patient, der dies nicht kann. Dies

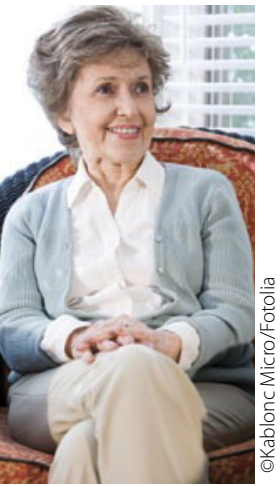

Gute Prognose! wurde in einer prospektiven Beobachtungsstudie einer Münchner Klinik deutlich.

34 der 120 Patienten, die wegen eines schweren Schlaganfalls stationär behandelt worden waren, schlugen bereits in den ersten Tagen nach dem Ereignis die Beine übereinander. Ihnen wurde eine Kontrollgruppe mit 34 Schlaganfallpatienten zugeordnet, die dies nicht konnten. Ein Jahr nach der Klinikentlassung waren Patienten, die die Beine übereinanderschlagen konnten, zwar etwas beeinträchtigt, konnten aber selbstständig laufen. Der durchschnittliche „Nichtübereinanderschlager" dagegen war schwer behindert und auf permanente Hilfe angewiesen. Nur einer der Patienten, die die Beine übereinanderschlugen, starb, in der unbeweglicheren Kontrollgruppe dagegen waren es 18.
\end{abstract}

